\title{
PREVALÊNCIA DE ASMA EM ESCOLARES DA REDE PÚBLICA DE ENSINO NO MUNICÍPIO DE URUGUAIANA/RS
}

\author{
PREVALENCE OF ASTHMA IN CHILDREN IN THE PUBLIC \\ SCHOOL SYSTEM IN THE MUNICIPALITY OF URUGUAIANA/RS
}

\section{Danize Aparecida Rizzetti', Guilherme de Freitas Teodósio², Renato Saraiva Juchem ${ }^{2}$, Giulia Alessandra Wiggers ${ }^{3}$}

\section{RESUMO}

Este estudo objetiva estimar a prevalência de sintomas de asma e de provável asma em escolares de Uruguaiana, estado do Rio Grande do Sul, e relacioná-los com possíveis fatores de risco. Para isso, participaram do estudo 391 crianças e adolescentes nas faixas etárias de 6-7 e 13-14 anos, tanto do gênero feminino quanto masculino, cujos responsáveis preencheram corretamente o questionário ISAAC fase I aplicado durante o período de janeiro a novembro de 2015. A prevalência de asma relacionada aos sintomas e fatores de risco na população escolar de Uruguaiana foi de $15 \%$, correspondendo $19 \%$ na faixa etária de 6-7 anos e $12 \%$ na faixa etária de 13-14 anos. Não houve relação estabelecida entre prevalência de sintomas asmáticos e fatores ambientais. Conclui-se que a prevalência de sintomas asmáticos e de provável asma é similar as outras cidades do RS.

Descritores: Asma; Escolares; Questionário ISAAC.
${ }^{1}$ Mestre em Bioquímica pela Universidade Federal do Pampa (UNIPAMPA), Uruguaiana, RS, Brasil.

2 Graduado em Fisioterapia pela Universidade Federal do Pampa (UNIPAMPA), Uruguaiana, RS, Brasil.

${ }^{3}$ Doutora em Ciências Fisiológicas pela Universidade Federal do Espírito Santo (UFES), Vitória, ES, Brasil

\begin{abstract}
This study aims to estimate the prevalence of asthma symptoms and likely asthma in children of Uruguaiana, State of Rio Grande do Sul, and relate them to possible risk factors. For this, participated of the study 391 children and adolescents in the age groups 6-7 and 13-14 years,

both female and male genres, whose leaders consented to the study and correctly completed the questionnaire ISAAC phase I applied during the period from March to December 2015. The prevalence of asthma and related symptoms in the school population of Uruguaiana was $15 \%$, representing $19 \%$ aged $6-7$ years and $12 \%$ aged $13-14$ years. There was no relationship established between the prevalence of asthma symptoms and environmental factors. It concludes that the prevalence of asthma symptoms and likely asthma is similar to other cities in the RS.
\end{abstract}

Descriptors: Asthma; School Population; ISAAC Questionary. 


\section{Introdução}

A asma é uma doença inflamatória crônica das vias aéreas inferiores, predominantemente eosinofílica, que resulta em obstrução recorrente do fluxo aéreo, reversível espontaneamente ou como resultado de terapia. Os sintomas recorrentes são dispneia, sibilância, tosse e dor torácica ${ }^{1,2}$. Dados epidemiológicos datados de 2014 apontam que aproximadamente 20 milhões de pessoas são asmáticas no Brasil ${ }^{3}$, sendo esta doença responsável por 5 a 10\% das mortes por causas respiratórias ${ }^{4,5}$. A maior incidência desta doença é na infância devido a fatores anatômicos, como 0 estreitamento das vias aéreas; fisiológicos, como a imaturidade do sistema imunológico; e ambientais, devido à exposição a fatores de risco desencadeantes das crises de hiperresponsividade brônquica ${ }^{6,7}$. No Brasil, a prevalência média de asma entre escolares e adolescentes, está entre 25,7\% e 21,4\%, respectivamente, com variações regionais ${ }^{8}$.

A investigação epidemiológica de doenças alérgicas do trato respiratório, bem como a prevalência e gravidade de asma para a população infantil pode ser realizada por meio do questionário derivado do Study of Asthma and Allergies in Childhood ${ }^{9}$, validado no Brasil ${ }^{10}$. Este instrumento permite a descrição da prevalência de asma, rinoconjuntivite alérgica e eczema atópico, sendo realizada em duas faixas etárias (6-7 e 13-14 anos de idade). 0 estudo original, realizado em 56 países demonstrou variabilidade de asma ativa nos países estudados de $1,6 \%$ a $36,8 \%{ }^{9}$. O Brasil situou-se em oitavo lugar, com a prevalência de $20 \%{ }^{11}$. Nas sete cidades brasileiras participantes do estudo original, a prevalência de asma nas faixas etárias de 6 a 7 e 13 a 14 anos foram: São Paulo (6,1\% e 10\%), Curitiba (6,6\% e 8,6\%), Porto Alegre (16,8\% e $21,9 \%)$, Recife $(20,7 \%$ e $21,0 \%)$, Uberlândia $(5,4 \%$ e $15,1 \%)$, Itabira $(4,7 \%$ e $4,8 \%)$ e Salvador $(12,6 \%)^{10}$. Além de Porto Alegre, o estado do Rio Grande do Sul ampliou a avaliação em estudos posteriores para as cidades de Santo Ângelo $(22,6 \%)^{12}$, Passo Fundo $(31,2 \%)^{13}$, Rio Grande $(27,9 \%)^{14}$, Pelotas $(12,8 \%)^{15}$, Santa Maria $(16,7 \%)^{16}$, e ljuí $(19 \%)^{17}$.

A intensa variação térmica existente no estado do Rio Grande do Sul (RS), muitas vezes dentro de uma mesma região e estação climática do ano, principalmente em cidades localizadas na fronteira oeste, como Uruguaiana ${ }^{18}$, onde há a maior amplitude térmica do país, pode ser um fator importante para a ocorrência constante de sintomas de asma na população infantil. Assim, estudos epidemiológicos que identifiquem a prevalência de asma em crianças nesta região são essenciais. Nesse contexto, o objetivo deste estudo foi estimar a prevalência de sintomas asmáticos e de provável asma, assim como relacioná-los à exposição a possíveis fatores de risco pelos escolares do ensino fundamental das escolas de rede pública de ensino de Uruguaiana, RS.

\section{Metodologia}

Estudo do tipo observacional, com delineamento transversal descritivo, o qual obteve aprovação do Comitê de Ética em Pesquisa da instituição (CEP/UNIPAMPA-CAAE 34945214.2.0000.5323), e obedeceu ao constante na Resolução 466/12 do Conselho Nacional de Saúde. A seleção das escolas participantes do estudo foi realizada por meio de amostragem por Clusters, onde a cidade foi dividida em quadrantes, e, para cada um deles, estabeleceu-se o número de escolas e alunos. Foi realizado um sorteio entre as escolas de cada quadrante, mantendo a proporção de alunos por região. A visita às escolas foi realizada no período de janeiro a novembro de 2015 , de acordo com a ordem da sequência sorteada. Foi considerada representativa da população em estudo uma amostra igual ou superior a 377 estudantes, de acordo com a fórmula de determinação de amostra para população infinita, considerando um nível de confiança de $95 \%$ e um erro máximo de estimação de 3\% ${ }^{19}$.

Utilizou-se como instrumento de coleta de dados o questionário da fase I do estudo "The international study of asthma and allergies in childhood steering committe - ISAAC" 9 . Este questionário é estruturado, auto-aplicável e foi validado no Brasil por Solé et al. (1998) ${ }^{10}$ para aferir diferenças de prevalência de asma e rinite em várias regiões. Ele é um questionário com boa sensibilidade $(0,85)$ e especificidade $(0,81)$, sendo comparado ao diagnóstico de especialistas em pneumologia ${ }^{10}$. Possui 12 questões referentes aos sinais e sintomas de asma, alergias, fumo e bronquite e avalia a prevalência de asma em duas faixas pediátricas de grande importância para estas doenças, 6-7 anos e 13-14 anos. 0 questionário foi distribuído a todos os escolares que se encontravam nas faixas etárias estipuladas pelo ISAAC.

Foram incluídos os estudantes do ensino fundamental de escolas da rede pública de ensino da cidade de Uruguaiana que assinaram um Termo de Assentimento referente à participação na pesquisa e cujos pais ou responsáveis autorizaram a mesma através da assinatura do Termo de Consentimento Livre e Esclarecido (TCLE) e responderam de maneira completa e correta ao questionário. Foram excluídos aqueles estudantes que não retornaram aos pesquisadores o questionário ou que os tenham retornado com erros de preenchimento (perguntas com dupla marcação de alternativas ou sem marcação de resposta). 
Das 12 perguntas presentes no ISAAC fase I, quatro são referentes à ocorrência de sintomas de asma (sibilos alguma vez, sibilos no último ano, sibilos desencadeados pelo exercício e tosse seca noturna), três referem-se à gravidade dos sintomas (número de episódios ou crises de sibilos por ano, sibilância noturna e sibilância dificultando a fala) e uma pergunta é referente ao diagnóstico de asma. Para este estudo a determinação da prevalência de sintomas asmáticos baseou-se no protocolo descrito por Ferrari et al. $(1998)^{20}$, o qual estabelece como diagnóstico de provável asma a presença de uma das seguintes condições: quatro ou mais crises no último ano, ou com número menor de crises (uma a três) e sono interrompido por sibilância no último ano, ou sem interrupção do sono, porém com asma de exercício e tosse noturna. Para verificar a prevalência dos sintomas de asma entre os escolares de Uruguaiana, RS, bem como os fatores de risco relacionados a esta doença e a prevalência de casos de asma provável foi utilizada a estatística descritiva. Os dados foram apresentados por meio da porcentagem de respostas positivas às diferentes questões do questionário.

\section{Resultados}

Foram visitadas cinco escolas no município de Uruguaiana, sendo encontrado um total de 1.453 alunos elegíveis para o estudo. $O$ questionário foi distribuído a 1.335 escolares, com retorno de 520 (38\%), dos quais, 391 (75\%) foram incluídos no estudo. Os demais foram excluídos por não terem sido corretamente preenchidos (perguntas com dupla marcação de alternativas ou sem marcação de resposta). A tabela 1 apresenta as características dos escolares cujos questionários foram analisados. Dos 391 escolares participantes, 174 (44,5\%) eram do sexo masculino e 217 (55,5\%) do feminino. A tabela 2 mostra as frequências das respostas afirmativas dos escolares das duas faixas etárias sobre sintomas referentes à asma. Para as duas faixas etárias investigadas os sintomas mais prevalentes encontrados foram presença de sibilos nos últimos 12 meses (56\% para 6-7 anos e 37\% para 13-14 anos) e sibilância alguma vez na vida (55\% para 6-7 anos e 50\% para 13-14 anos), seguidos de tosse noturna sem resfriado (43\% para 6-7 anos e 33\% para 13-14 anos).

Tabela 1 - Distribuição dos escolares por sexo e faixa etária

\begin{tabular}{c|c|c|c|c|c|c}
\hline \multirow{2}{*}{ Escolares } & \multicolumn{2}{|c|}{ Total } & \multicolumn{2}{c|}{ Masculino } & \multicolumn{2}{c}{ Feminino } \\
\cline { 2 - 7 } & $\mathrm{N}$ & $\%$ & $\mathrm{~N}$ & $\%$ & $\mathrm{~N}$ & $\%$ \\
\hline 6-7 anos & 161 & 41,2 & 58 & 36,0 & 103 & 64,0 \\
\hline 13-14 anos & 230 & 58,8 & 116 & 50,4 & 114 & 49,6 \\
\hline
\end{tabular}

Tabela 2 - Prevalência, em porcentagem, de sintomas de asma nos escolares de acordo com o sexo e grupo etário

\begin{tabular}{l|c|c|c|c|c|c}
\hline \multicolumn{2}{c|}{ Questão } & \multicolumn{7}{c}{$\mathbf{6 - 7}$ anos (N=161) } \\
\cline { 2 - 8 } & $\mathbf{M}(\mathbf{n = 5 8 )}$ & $\%$ & $\mathbf{F}(\mathbf{n = 1 0 3 )}$ & $\%$ & Subtotal & $\%$ \\
\hline Sibilos alguma vez na vida & 33 & 37 & 56 & 63 & 89 & 55 \\
\hline Sibilos nos últimos 12 meses & 25 & 28 & 65 & 72 & 90 & 56 \\
\hline Frequência das crises de sibilos no último ano & 22 & 44 & 28 & 52 & 50 & 31 \\
\hline Uma a três crises & 1 & 2 & 6 & 6 & 7 & 4 \\
\hline
\end{tabular}

\begin{tabular}{c|c|c|c|c|c|c}
\hline Frequência dos sibilos durante a noite no último ano \\
\hline Acordou > de 1 noite por semana & 13 & 52 & 12 & 48 & 25 & 16 \\
\hline Acordou < de 1 noite por semana & 7 & 28 & 18 & 72 & 25 & 16 \\
\hline $\begin{array}{c}\text { Sibilo forte dificultando a fala } \\
\text { no último ano }\end{array}$ & 6 & 6 & 4 & 40 & 10 & 6 \\
\hline Asma ou bronquite alguma vez na vida & 20 & 32 & 42 & 68 & 62 & 39 \\
\hline Sibilos pós-exercícios & 9 & 53 & 8 & 47 & 17 & 11 \\
\hline Tosse noturna sem resfriado & 24 & 35 & 45 & 65 & 69 & 43 \\
\hline
\end{tabular}




\begin{tabular}{c|c|c|c|c|c|c|c|c}
\hline \multirow{2}{*}{ Questão } & \multicolumn{4}{|c|}{ 13-14 anos (N=230) } & \multirow{2}{*}{$\begin{array}{c}\text { Total } \\
(\mathbf{N}=391)\end{array}$} & \multirow{2}{*}{} \\
\cline { 2 - 11 } & M (n=116) & \% & $\mathbf{F ~ ( n = 1 1 4 ) ~}$ & \% & Subtotal & \% & \\
\hline Sibilos alguma vez na vida & 51 & 49 & 54 & 51 & 105 & 46 & 194 & 50 \\
\hline Sibilos nos últimos 12 meses & 27 & 50 & 27 & 50 & 54 & 23 & 144 & 37 \\
\hline
\end{tabular}

Frequência das crises de sibilos no último ano

\begin{tabular}{c|c|c|c|c|c|c|c|c}
\hline Uma a três crises & 19 & 45 & 23 & 55 & 42 & 18 & 92 & 23.5 \\
\hline Mais de quatro crises & 3 & 60 & 2 & 40 & 5 & 2 & 12 & 3 \\
\hline
\end{tabular}

Frequência dos sibilos durante a noite no último ano

\begin{tabular}{c|c|c|c|c|c|c|c|c}
\hline Acordou > de 1 noite por semana & 23 & 66 & 12 & 34 & 35 & 15 & 60 & 15 \\
\hline Acordou < de 1 noite por semana & 3 & 33 & 6 & 67 & 9 & 4 & 34 & 9 \\
\hline $\begin{array}{c}\text { Sibilo forte dificultando a fala } \\
\text { no último ano }\end{array}$ & 8 & 67 & 4 & 33 & 12 & 5 & 22 & 6 \\
\hline $\begin{array}{c}\text { Asma ou bronquite alguma } \\
\text { vez na vida }\end{array}$ & 32 & 47 & 36 & 53 & 68 & 30 & 130 & 33 \\
\hline Sibilos pós-exercícios & 13 & 50 & 13 & 50 & 26 & 11 & 43 & 11 \\
\hline Tosse noturna sem resfriado & 25 & 42 & 35 & 58 & 60 & 26 & 129 & 33 \\
\hline
\end{tabular}

Ao se investigar os casos de provável asma entre os escolares, por meio da presença dos sintomas - quatro ou mais crises no último ano ou número menor de crises (uma a três) e sono interrompido por sibilância no último ano; ou sem interrupção do sono, porém asma de exercício e tosse noturna - verificou-se a prevalência de provável asma em $55(14 \%)$ dos escolares avaliados, onde 27 (21\%) escolares compreendidos na faixa etária de 6-7 anos e 28 (12\%) na faixa etária de 13-14 anos, conforme demonstra a tabela 3.

Tabela 3 - Prevalência (\%) de diagnóstico de provável asma entre os escolares participantes

\begin{tabular}{c|c|c}
\hline Questões para diagnóstico de provável asma & $\begin{array}{c}\text { Prevalência } \\
\text { (\%) } \\
\mathbf{6 - 7} \text { anos }\end{array}$ & $\begin{array}{c}\text { Prevalência } \\
\text { (\%) } \\
\mathbf{1 3 - 1 4} \text { anos }\end{array}$ \\
\cline { 2 - 3 }$(\mathrm{N}=161)$ & $7 \%(4)$ & $2 \%(5)$ \\
\hline 4 ou mais crises nos últimos 12 meses & $10 \%(16)$ & $7 \%(17)$ \\
\hline 1 a 3 crises e sono interrompido por sibilos & $4 \%(7)$ & $3 \%(6)$ \\
\hline $\begin{array}{c}1 \text { a } 3 \text { crises, sem alterar sono, porém com sibilos } \\
\text { pós-exercícios físicos e tosse noturna }\end{array}$ & $21 \%(27)$ & $12 \%(28)$ \\
\hline TOTAL
\end{tabular}

Com relação aos fatores de risco para asma investigados, foram analisados o convívio com fumantes no mesmo domicílio e a exposição a animais domésticos pelos escolares de ambas as faixas etárias. Para a faixa etária de 6-7 anos, a maior parte deles, ou seja, 95 (59\%) escolares, não convivem com fumantes em suas residências, porém em sua maioria, 124 (77\%) estudantes possuem animal de estimação em casa. 0 mesmo ocorre para a faixa etária de 13-14 anos, onde também se observa que a maior parte dos escolares, 169 (73\%) estudantes não convive com fumantes em suas residências, porém 176 (76,5\%) deles possuem animal de estimação em sua residência.

O diagnóstico de provável asma foi verificado em $13(20 \%)$ dos escolares na faixa etária de 6-7 anos que convivem com fumantes, e 5 (8\%) na faixa etária de 13-14 anos. Já para os estudantes que convivem com animais de estimação em suas residências $24(19 \%)$ apresentaram provável diagnóstico de asma na faixa etária de 6-7 anos e 20 (11\%) na faixa etária de 13-14 anos (tabelas 4 e 5). 
Tabela 4 - Prevalência (\%) de diagnóstico de provável asma entre os escolares de acordo com o fator de risco convívio com fumantes

\begin{tabular}{c|c|c|c|c}
\hline $\begin{array}{c}\text { Questões para } \\
\text { diagnóstico de provável } \\
\text { asma } \\
(\mathbf{N}=391)\end{array}$ & $\begin{array}{c}\text { Com convívio } \\
\text { com fumante } \\
(41 \%)(n=66)\end{array}$ & $\begin{array}{c}\text { Sem convívio } \\
\text { com fumante } \\
(59 \%)(n=95)\end{array}$ & $\begin{array}{c}\text { Com convívio } \\
\text { com fumante } \\
(17 \%)(n=61)\end{array}$ & $\begin{array}{c}\text { Sem convívio } \\
\text { com fumante } \\
(73 \%)(n=169)\end{array}$ \\
\cline { 2 - 5 } & $3 \%(2)$ & $5 \%(5)$ & $3 \%(2)$ & $2 \%(3)$ \\
\hline $\begin{array}{c}4 \text { ou mais crises nos } \\
\text { últimos } 12 \text { meses }\end{array}$ & $12 \%(8)$ & $8 \%(8)$ & $5 \%(3)$ & $8 \%(12)$ \\
\hline $\begin{array}{c}1 \text { a } 3 \text { crises e sono } \\
\text { interrompido por sibilos }\end{array}$ & $4,5 \%(3)$ & $4 \%(4)$ & $0 \%(0)$ & $3,5 \%(6)$ \\
\hline $\begin{array}{c}1 \text { a } 3 \text { crises, sem alterar } \\
\text { sono, porém com sibilos } \\
\text { pós-exercícios físicos e } \\
\text { tosse noturna }\end{array}$ & & & & \\
\hline & & $17 \%(17)$ & & $14 \%(21)$ \\
\hline
\end{tabular}

Tabela 5 - Prevalência (\%) de diagnóstico de provável asma entre os escolares de acordo com o fator de risco convívio com animais domésticos

\begin{tabular}{|c|c|c|c|c|}
\hline \multirow{2}{*}{$\begin{array}{c}\text { Questões para } \\
\text { diagnóstico de provável } \\
\text { asma } \\
(\mathbf{N}=391)\end{array}$} & \multicolumn{2}{|c|}{ 6-7 anos ( $N=161)$} & \multicolumn{2}{|c|}{ 13-14 anos $(\mathrm{N}=230)$} \\
\hline & $\begin{array}{c}\text { Com convívio } \\
\text { com animais } \\
(77 \%) \\
(n=124)\end{array}$ & $\begin{array}{c}\text { Sem convívio } \\
\text { com animais } \\
(23 \%) \\
(\mathrm{n}=37)\end{array}$ & $\begin{array}{l}\text { Com convívio } \\
\text { com animais } \\
(76.5 \%)(n=176)\end{array}$ & $\begin{array}{l}\text { Sem convívio } \\
\text { com animais } \\
(23.5 \%)(n=54)\end{array}$ \\
\hline $\begin{array}{l}4 \text { ou mais crises nos } \\
\text { últimos } 12 \text { meses }\end{array}$ & $5 \%(6)$ & $3 \%(1)$ & $2 \%(4)$ & $2 \%(1)$ \\
\hline $\begin{array}{l}1 \text { a } 3 \text { crises e sono } \\
\text { interrompido por sibilos }\end{array}$ & $11 \%(14)$ & $5 \%(2)$ & $7 \%(12)$ & $9 \%(5)$ \\
\hline $\begin{array}{c}1 \text { a } 3 \text { crises, sem alterar } \\
\text { sono, porém com sibilos } \\
\text { pós exercícios físicos e } \\
\text { tosse noturna }\end{array}$ & $3 \%(4)$ & $8 \%(2)$ & $2 \%(4)$ & $4 \%(2)$ \\
\hline TOTAL & $19 \%(24)$ & $16 \%(5)$ & $11 \%(20)$ & $15 \%(8)$ \\
\hline
\end{tabular}

\section{Discussão}

O município de Uruguaiana está localizado no extremo oeste do estado do RS. A principal característica climática do município é a grande amplitude térmica, sendo a maior do nosso país, com estações bem definidas, sendo o verão muito quente e o inverno extremamente frio, com temperaturas negativas, geada e nevoeiro ${ }^{18,21}$. As alterações de temperatura são fatores predisponentes e desencadeantes de doenças respiratórias ${ }^{22}$. No entanto, a incidência de diagnóstico de provável asma no município de Uruguaiana avaliada neste estudo é similar a outras regiões do Brasil e do RS.

Os resultados encontrados neste estudo reuniram informações sobre a prevalência dos sintomas e provável diagnóstico da asma, associando com possíveis fatores de risco em uma população de crianças e adolescentes das escolas da rede pública do município de Uruguaiana, RS. Foram encontradas 55 (14\%) crianças com provável diagnóstico de asma, sendo que dessas, 27 (21\%) estavam compreendidas na faixa etária entre 6-7 anos e $28(12 \%)$ na faixa etária entre 13-14 anos. Estudos envolvendo um total de 40.111 adolescentes de 13 a 14 anos em 15 cidades brasileiras participantes do estudo ISAAC fase I demonstraram uma variação na prevalência de asma atual de $6 \%$ a $26,4 \%$, com uma média nacional de $21,4 \%{ }^{10}$. Já para 0 diagnóstico médico de asma, a média foi de 14,3\%. Nesse contexto, portanto, o município de Uruguaiana, RS, está na média de prevalência dos municípios brasileiros estudados e também se enquadra na média das cidades gaúchas participantes. 
Comparando-se a prevalência de asma nas diferentes cidades brasileiras que realizaram o estudo ISAAC, percebe-se que São Paulo (SP) ${ }^{23}$, Duque de Caxias (RJ) ${ }^{24}$ e Porto Alegre (RS) $)^{25}$ obtiveram o maior índice na prevalência de asma provável em escolares de 6 a 14 anos. Os fatores ambientais que atuam sobre a asma, aumentando as suas taxas de morbidade e gravidade mais importantes são os poluentes no ar atmosférico, tanto externo quanto interno, e as condições meteorológicas do local, como a temperatura, a umidade relativa do ar e a velocidade dos ventos, que podem aumentar a duração da exposição aos poluentes e impedir sua dissipação $0^{26,27}$. O município de Uruguaiana não apresenta estas características industriais semelhantes às cidades de maior índice na prevalência de asma, muito embora apresente grandes alterações climáticas e amplitude térmica durante suas estações ou até mesmo no período de 24 horas, o que poderia aumentar a incidência de asma. No entanto, não encontramos índices maiores em Uruguaiana o que não faz nexo causal entre as grandes alterações climáticas e a prevalência de asma.

Dos 1.335 escolares que receberam o questionário apenas 520 (38\%) retornaram as respostas, dos quais, 391 (75\%) foram incluídos no estudo. Esta alta taxa de não retorno também foi relatada em estudos similares que atribuem este fato ao baixo índice de alfabetização dos pais, medo de assinar o termo de consentimento ou desconfiança em relação às implicações do estudo, falta de contato direto entre os entrevistadores e os pais que eram os responsáveis pelo preenchimento dos questionários, pois apenas a carta enviada pode não ter sido suficiente para sensibilizar e convencer alguns pais da importância do estudo ${ }^{28}$. Mesmo sendo um questionário de fácil entendimento, para alguns autores, o fato de sua aplicação não depender da presença de um entrevistador no local contribui para um significativo índice de erro no seu preenchimento, acarretando níveis elevados de exclusão de amostra nos estudos ${ }^{15,29}$.

A prevalência de provável asma encontrada neste estudo, a qual foi de $21 \%$ para faixa etária de 6-7 anos e 12\% para faixa etária de 13-14 anos seguiu os critérios propostos por Ferrari et al. (1998) ${ }^{20}$. Para este autor, as perguntas presentes no questionário não seriam suficientes para um diagnóstico real quando analisadas de maneira isolada, assim estabeleceu como critérios para diagnóstico de provável asma uma análise conjunta das informações sobre as crianças ou adolescentes com 4 ou mais crises no último ano, ou com número menor de crises (1 a 3), e sono interrompido por sibilância no último ano, ou sem interrupção do sono, porém com asma de exercício e tosse noturna. O presente estudo considerou as mesmas condições definidas pelo autor acima mencionado para a detecção de um provável caso de asma, de acordo com as características clínicas da doença. Em relação às demais cidades do Rio Grande do Sul, que realizaram a pesquisa, Uruguaiana encontra-se semelhante a outros municípios. Na faixa etária de 6-7 anos Uruguaiana apresenta a prevalência similar à cidade de ljuí $(19 \%)^{17}$. Já em relação a faixa etária de 13-14 anos a prevalência de provável diagnóstico de asma está dento da média estadual e próxima da prevalência de Pelotas $(12,8 \%)^{15}$.

Não foi observada a prevalência de sintomas asmáticos em escolares expostos a fatores ambientais, como fumaça de cigarro e pêlos de animais domésticos. O mecanismo pelo qual o tabagista passivo aumenta os sintomas respiratórios e diminui a função pulmonar em criança não é bem esclarecido ${ }^{22}$. Em contrapartida, um estudo de coorte com 440 crianças com história familiar de atopia em Boston (USA) mostrou que a exposição passiva à fumaça de cigarro tem sido relacionada à doença sibilante nos primeiros anos de vida e à asma na idade escolar ${ }^{27}$.

Animais domésticos peludos como cães e gatos podem causar ou agravar doenças respiratórias. Estudo realizado com lactentes do sul do Brasil demonstrou que a presença de cão e outros animais domésticos no domicílio durante a gestação foi um fator de risco para a presença de sibilância na população estudada ${ }^{30}$. Diferentemente disso, neste estudo, não foi encontrada uma associação entre exposição a cães e gatos e asma, resultado semelhante ao encontrado em estudo desenvolvido com 561 escolares, provenientes de 35 escolas públicas da cidade de São Paulo ${ }^{11}$. A associação entre a exposição a animais de estimação e o risco de asma têm sido difícil de determinar, principalmente pelas diferentes metodologias adotadas nos estudos, já que esta relação está muito dependente da duração e da intensidade da exposição ao animal, bem como da espécie do animal a que a criança é exposta, detalhes que muitas vezes são difíceis de analisar neste tipo de estudo.

Quanto às limitações do estudo, o baixo índice de retorno de questionários corretamente preenchidos pode ter influenciado os resultados encontrados, tendo em vista que, de maneira informal, os professores das escolas visitadas relataram que esperavam um índice superior de alunos com provável diagnóstico de asma. Para estudos futuros, sugere-se a realização de uma palestra informativa aos pais ou responsáveis pelos escolares com o objetivo de prestar orientações para o correto preenchimento do questionário. 


\section{Considerações Finais}

A partir do presente estudo, concluímos que, muito embora o município de Uruguaiana, $R S$, apresente características climáticas peculiares que poderiam influenciar a ocorrência e a gravidade de doenças respiratórias crônicas, principalmente na população infantil, a prevalência de provável asma em escolares da cidade foi similar às encontradas em outros municípios do estado e se enquadra dentro da média nacional. Não se observou uma prevalência de sintomas asmáticos ou asma provável em escolares expostos a condições de risco, como convívio com fumantes e exposição a animais domésticos. Estes resultados poderão ser utilizados em futuros estudos epidemiológicos, objetivando um conhecimento mais abrangente da evolução da asma e a compreensão dos fenômenos biológicos relacionados, permitindo elaboração de medidas de prevenção eficazes para o controle desta doença.

\section{Referências}

1. Sociedade Brasileira de Pneumologia e Tisiologia. III Consenso Brasileiro no Manejo da Asma. J Pneumol. 2002; 28:S4-5.

2. Global Initiative for Asthma [Internet]. Available from <http://www.ginasthma.com>. [Access on january 2, 2015].

3. Brasil. Departamento de Informática do SUS - DATASUS [Internet]. Available from <http://www2.datasus.gov.br>. [Access on january 3, 2015].

4. Silva ECF. Asma Brônquica. Revista Hospital Universitário Pedro Ernesto. 2008; v.7.

5. Brasil. Ministério da Saúde [Internet]. Available from <http://bvsms.saude.gov.br>. [Access on january 3, 2015].

6. Celedon JC, Milton DK, Ramsey CD, Litonjua AA, Ryan L, Platts-Mills TAE, et al. Exposure to dust mite allergen and endotoxin in early life and asthma and atopy in childhood. J Allergy Clin Immunol. 2007; 120:144-9.

7. World Health Organization [Internet]. Available from <http://www.who.int>. [Access on december 14, 2014].

8. Solé D, Camelo ICN, Wandalsen GF, Naspitz CK, Vanna AT, Amorim A, et al. A asma em crianças é um problema de saúde pública? Rev Bras Alergia Imunopatol. 2004; 27:185-8.

9. International Study of Asthma and Allergies in Childhood (ISAAC) Steering Committee. ISAAC - The International Study of Asthma and Allergies in Childhood: Background and methods. Eur Respir J. 1996; 9:410.

10. Solé D, Vanna AT, Yamanda E, Rizzo MC, Naspitz CK. International Study of Asthma and Allergies in Childhood (ISAAC) written questionnaire: validation of the asthma component among Brazilian children. J Invest Allergol Clin Immunol. 1998; 8:376-82.

11. Solé D. Prevalência e mortalidade por asma na cidade de São Paulo. [Doctoral Thesis]. Universidade de São Paulo (USP). Programa de Pós-Graduação em Medicina. São Paulo; 1997.

12. Fenner AP, Lazzari JH. Prevalência de asma e rinite alérgica em escolares no município de Santo Ângelo/RS. Revista da AMRIGS. 2009; 53:122-7.

13. Neto ACP. Fatores de risco para a asma e rinite alérgica em população de escolares na cidade de Passo Fundo, RS. [Doctoral Thesis]. Universidade Federal do Rio Grande do Sul (UFRGS). Programa de Pós-Graduação em Saúde da Criança e do Adolescente. Porto Alegre; 2012.

14. Prietsch SOM. Sibilância recorrente em uma coorte de crianças menores de 13 anos, em Rio Grande, RS. [Doctoral Thesis]. Universidade Federal do Rio Grande do Sul (UFRGS). Programa de Pós-Graduação em Medicina. Porto Alegre; 2005.

15. Chatkin MN, Menezes AMB. Prevalência e fatores de risco para asma em escolares de uma coorte no Sul do Brasil. J Pediatr. 2005; 81:411-6.

16. Cassol VE, Solé D. Prevalência de asma em adolescentes urbanos de Santa Maria (RS). Projeto ISAAC- International Study of Asthma and Allergies in childhood. J Bras Pneumol. 2005; 31:191-6.

17. Strassburger SZ. Impacto da asma na qualidade de vida em escolares do município de ijuí/RS. [Doctoral Thesis]. Pontifícia Universidade Católica do Rio Grande do Sul. (PUCRS). Programa de Pós-Graduação em Medicina Pediatria e Saúde da Criança. Porto Alegre; 2014.

18. Brasil. Instituto Nacional de Meteorologia - INMET [Internet]. Available from <http://www.inmet.gov.br>. [Access on december 20, 2014].

19. Dawson B, Trapp RG. Bioestatística Básica e Clínica. McGraw-Hill, 3rd ed, 2001.

20. Ferrari FP, Rosário Filho NA, Ribas LFO, Callefe LG. Prevalência de asma em escolares de Curitiba - Projeto ISAAC. J Pediatr. 1998; 74:299-305. 
21. Brasil. Instituto Brasileiro de Geografia e Estatística - IBGE [Internet]. Available from <http://www.ibge.gov.br>. [Access on january 8, 2015].

22. Brunekreef B, Groot B, Hoek G. Pets, allergy and respiratory symptoms in children. Int J Epidemiol. 1992; 21:338-42.

23. Casagrande RRD, Pastorino AC, Souza RGL, Leone C, Solé D, Jacob CMA. Prevalência de asma e fatores de risco em escolares da cidade de São Paulo. Rev Saúde Pública. 2008; 42:517-23.

24. Boechat JL, Rios JL, Sant'anna CC, França AT. Prevalência e gravidade de sintomas relacionados à asma em escolares e adolescentes no município de Duque de Caxias, Rio de Janeiro. J Bras Pneumol. 2005; 31:111-7.

25. Fiore RW, Comparsi AB, Reck CL, Oliveira JK, Pampanelli KB, Fritscher CC. Variação na prevalência de asma e atopia em um grupo de escolares de Porto Alegre, Rio Grande do Sul. J Pneumol. 2001; 27:237-42.

26. Saldanha CT, Silva AMC, Botelho C. Variações climáticas e uso de serviços de saúde em crianças asmáticas menores de cinco anos de idade: um estudo ecológico. J Bras Pneumol. 2005; 31:492-8.

27. Arshad SH. Does exposure to indoor allergens contribute to the development of asthma and allergy? Curr Allergy Asthma Rep. 2010; 10:49-55.

28. Asher MI, Keil U, Anderson HR, Beasley R, Crane J, Martinez F, et al. International Study of Asthma and Allergies in Childhood (ISAAC): Rationale and methods. Eur Respir J. 1995; 8:483-91.

29. Luna MFG, Fischer GB. Comparação temporal das prevalências de asma e rinite em adolescentes em Fortaleza, Brasil. J Bras Pneumol. 2013; 39:128-37.

30. Chong Neto HJ, Rosário NA. Risk factors for wheezing in the first year of life J Pediatr. 2008; 84:495-502.

\author{
Danize Aparecida Rizzetti \\ Endereço para correspondência - Universidade Federal do Pampa - Campus Uruguaiana \\ Rua: BR 472, Km 592, Cx. 118, Bairro: Saída Barra do Quaraí, \\ CEP: 97500-970, Uruguaiana, RS, Brasil. \\ E-mail: danize.rizzetti@gmail.com \\ Lattes: http://lattes.cnpq.br/0163307776523169 \\ Guilherme de Freitas Teodósio - guteodosio@hotmail.com \\ Renato Saraiva Juchem - renatojuchem@hotmail.com \\ Giulia Alessandra Wiggers - giuliapecanha@unipampa.edu.br
}

\title{
Enviado em 06 de novembro de 2015. Aceito em 01 de abril de 2016.
}


